\title{
Dinâmica sucessional de comunidade de algas perifíticas em um ecossistema lótico subtropical
}

\author{
Successional dynamic of the periphytic algal community in ecossistem lotic subtropical
}

\author{
Sirlene Aparecida Felisberto ${ }^{1,3}$ \& Liliana Rodrigues ${ }^{2}$
}

\begin{abstract}
Resumo
O principal objetivo deste trabalho foi descrever as mudanças na estrutura de comunidades de algas perifiticas durante o processo de colonização e sucessão, em período chuvoso, indicando suas espécies descritoras. O local selecionado para

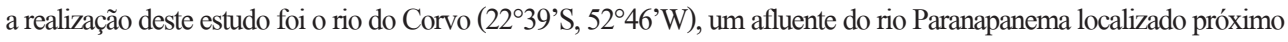
à barragem do reservatório de Rosana, na região noroeste do Paraná, Brasil. Coletas do perifiton em substrato artificial e medições de dados abióticos foram realizadas no período de 21 de novembro a 12 de dezembro de 2003 , emintervalos de três dias. A identificação e quantificação das algas foi feita em microscópio invertido, utilizando-se o método de Utermöhl. Para análise da dinâmica sucessional, foram considerados: riqueza de espécies, densidade total, abundância relativa dos táxons, espécies descritoras, diversidade de Shannon-Wiener e equitabilidade. As flutuações observadas na densidade total foram determinadas principalmente por Bacillariophyceae, Chlorophyceae, Cyanophyceae eZygnemaphyceae. Maiores valores de abundância na comunidade de algas perifiticas foram registrados para espécies pequenas de diatomáceas, abundantes durante todo o período, consideradas colonizadores eficientes e de crescimento rápido, assim como para cianobactérias e algas verdes filamentosas (Oedogonium spp. e Stigeoclonium spp.), de crescimento mais lento e mais abundantes nos estádios tardios. As primeiras estão entre as $r$-estrategistas, enquanto as últimas estão entre as C-S estrategistas (algas mais competitivas em sistemas mesotróficos e estáveis). A diversidade de Shannon-Wiener foi elevada (2,2 a 3,1), com o maior valor constatado na terceira semana de desenvolvimento sucessional. Com base em análise de componentes principais, as concentrações de nutrientes, o pH e a turbidez foram os principais fatores abióticos relacionados ao processo de sucessão. Palavras-chave: diversidade, estrutura da comunidade, perifiton, rio, sucessão.
\end{abstract}

\begin{abstract}
This paper aimed to describe the successional changes in the structure of periphytic algal assemblages in a lotic environment, during the rainy period, indicating the descriptor species. The study site was Corvo stream (22³9'S, $\left.52^{\circ} 46^{\prime} \mathrm{W}\right)$, a tributary of the Paranapanema river, located near the Rosana Reservoir, in the northwestern Paraná state, Brazil. Periphyton samples on artificial substrate and abiotic data were obtained from 21 November to 12 December 2003, at three-days intervals. Algae were identified and quantified using inverted microscope at 400X, according to Utermöhl. The analysis of the succession dynamics considered the following parameters: species richness, total density, relative abundance of taxa, descriptor species, Shannon-Wiener diversity and evennes. Fluctuations in total density were determined mainly by Bacillariophyceae, Chlorophyceae, Cyanophyceae, and Zygnemaphyceae. Higher values of abundance of the periphytic algae were recorded for small species of diatoms, known as efficient colonizers, which were abundant throughout the study period and showed rapid growth, as well as for Cyanobacteria and filamentous green algae (Oedogonium spp. and Stigeoclonium spp.), which were more abundant in the latter successional stages and showed slower growth. The first ones are among the r-strategists, while the latter are among the C-S strategists (more competitive algae in mesotrophic and stable systems). Shannon-Wiener diversity was high (2.2 to 3.1), with the highest value in the third week of study. According to the principal components analysis, nutrients concentrations, $\mathrm{pH}$ and turbidity were the most important abiotic factors for explaining the community structure changes throughout the succession. Key words: community structure, diversity, periphyton, river, succession.
\end{abstract}

${ }^{1}$ Universidade Federal de Goiás, Instituto de Ciências Biológicas, Depto. Biologia Geral, Programa de Pós-graduação em Ecologia e Evolução, Campus Samambaia, C.P. 131, 74001-970, Goiânia, GO, Brasil.

${ }^{2}$ Universidade Estadual de Maringá, Programa de Pós-graduação em Ecologia de Ambientes Aquáticos Continentais, Núcleo de Pesquisas em Limnologia, Ictiologia e Aqüicultura, bl. G-90, Av. Colombo 5790, 87020-900, Maringá, PR, Brasil.

${ }^{3}$ Autor para correspondência: sirfe@hotmail.com 


\section{Introdução}

A sucessão ecológica é entendida como a colonização sucessiva e contínua de um local por certas populações de espécies, acompanhada da extinção de outras (Townsend et al. 2010). $\mathrm{O}$ processo de sucessão pode culminar em um ecossistema estável, quando características estruturais da comunidade apresentarem valores máximos e fluxo contínuo (Odum 1969). Contudo, comunidades em equilíbrio podem se desestruturar devido a perturbações no ambiente, as quais tendem a provocar espaços "novos" nos quais as espécies serão redirecionadas, competindo por espaço e recursos (Odum 1969).

Para comunidade de algas perifíticas, na fase inicial de sucessão, ocorre a formação de uma camada orgânica, seguida da instalação de bactérias em poucas horas, além de diatomáceas oportunistas. Diatomáceas maiores colonizam o substrato em período de um dia a algumas semanas. Por fim, há a colonização por algas verdes (Hoagland et al. 1982, 1986; Biggs 1996). As espécies pioneiras serão aquelas que possuem propágulos abundantes e disponíveis na água, no local e no momento em que as superfícies estão disponíveis para a colonização (Biggs 1996; Stevenson 1996), podendo ser classificadas como $r$-estrategistas. Estas espécies pioneiras facilitam a invasão do substrato por outras espécies, podendo também persistir até serem substituídas por um competidor mais apto (Odum 1988; Biggs et al. 1998).

No modelo conceitual de matriz de hábitat construído por Biggs et al. (1998), as estratégias de algas perifíticas de ecossistemas lóticos temperados são: estrategistas competitivas (C), algas mais competitivas em sistemas eutróficos e estáveis; estrategistas C-S, algas mais competitivas em sistemas mesotróficos e estáveis; estrategistas tolerantes ao estresse (S), algas mais competitivas em sistemas oligotróficos e estáveis; estrategistas ruderais (R), algas mais competitivas em sistemas mesotróficos e com distúrbios frequentes. Com este modelo, seria possível predizer a biomassa do perifíton e os grupos funcionais de acordo com as condições dos ambientes em termos de distúrbios e recursos.

Dentre as variáveis ambientais, importantes no processo de sucessão do perifíton, destacamse a velocidade de corrente, que pode afetar a dominância, resistência e resiliência das espécies (McCormick \& Stevenson 1991; Peterson \&
Stevenson 1992) além de luz, que pode definir o padrão de recolonização em ecossistemas lóticos (Steinman et al. 1991). Vários estudos demonstraram a influência da variabilidade ambiental sobre a organização da comunidade (Stevenson 1996; Cetto et al. 2004; Vercellino \& Bicudo 2006; Borges \& Necchi Jr. 2008; Felisberto \& Rodrigues 2010).

Para ecossistema lótico brasileiro, Rodrigues \& Bicudo $(2001,2004)$ registraram que a maior contribuição relativa das algas perifíticas em processo sucessional pertenceu às diatomáceas. Branco et al. (2005) e Borges \& Necchi Jr. (2008), além de mostrarem a importância da disponibilidade de fósforo, revelaram que, sob influência da precipitação, as formas de crescimento das espécies de macroalgas alteraram-se em relação aos estádios iniciais e tardios.

As diatomáceas foram as algas dominantes (mais de $80 \%$ em abundância, comparados aos demais grupos de algas) na região lacustre do reservatório de Rosana (Felisberto \& Rodrigues 2005), no Paraná. Com base nesse dado, este estudo levantou a hipótese que, no rio do Corvo, tributário do reservatório de Rosana, o processo sucessional iniciará com diatomáceas de dimensões menores, principalmente de espécies $r$-estrategistas.

Considerando a importância do maior entendimento do processo de sucessão em ecossistemas lóticos subtropicais, o presente estudo tem por objetivo: a) descrever as mudanças na estrutura da comunidade de algas perifíticas em intervalos curtos (três dias), durante período de 21 dias; b) comparar a sequência de espécies abundantes e descritoras encontrada nas comunidades estudadas com o modelo de matriz conceitual para ecossistema lótico proposto por Biggs et al. (1998); e, c) identificar os principais fatores abióticos relacionados ao processo sucessional.

\section{Material e Métodos}

Área de Estudo

O local selecionado para a realização do presente estudo foi o rio do Corvo, localizado a aproximadamente $5 \mathrm{~km}$ da região lacustre do reservatório de Rosana ( $22^{\circ} 39^{\prime} \mathrm{S}$ e $52^{\circ} 46^{\prime} \mathrm{W}$, Fig. 1). O rio do Corvo é um dos tributários da margem esquerda deste reservatório, que está localizado no trecho inferior do rio Paranapanema, município de Diamante do Norte, estado do Paraná.

$\mathrm{O}$ rio apresenta cerca de $300 \mathrm{~m}$ de largura e $6 \mathrm{~m}$ de profundidade máxima. As margens, 


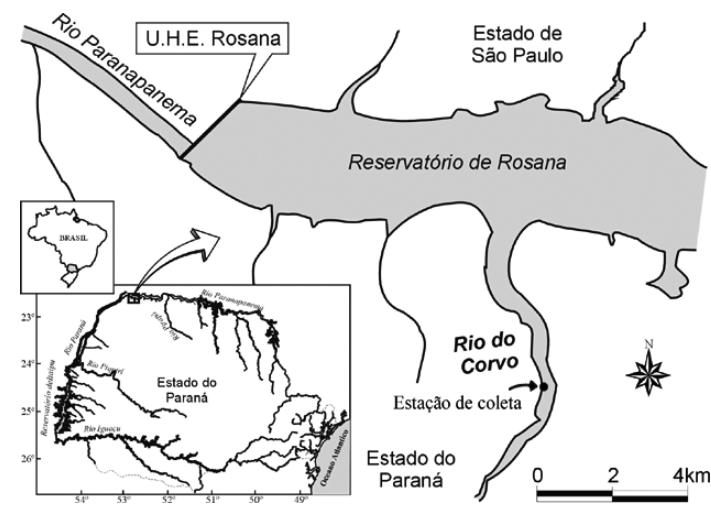

Figura 1- Área de estudo: reservatório de Rosana (Bacia do rio Paranapanema), no estado do Paraná, Brasil, com a localização da estação de coleta no rio do Corvo. Figure 1 - Study site: Rosana reservoir (Paranapanema river basin), Paraná state, Brazil, showing the sampling station in Corvo stream.

praticamente desprovidas de vegetação arbórea, apresentaram diversas macrófitas emersas (Typha sp., Sagittaria sp. e Eichhornia azurea (Sw.) Kunth.), flutuantes (Eichhornia crassipes (Mart.) Solms, Nymphaea sp. e Salvinia sp.) e submersas enraizadas (Cabomba furcata Schult. \& Schult., Egeria densa Planch., Egeria najas Planch., Myriophyllum sp. e Utricularia foliosa L.).

\section{Amostragem e análise dos dados}

A sucessão na comunidade perifítica foi acompanhada por 21 dias, entre 21 de novembro e 12 de dezembro de 2003, em intervalos de três dias. A primeira amostragem, correspondente ao terceiro dia de sucessão, ocorreu em 24 de novembro de 2003.

O clima na área de estudo, considerado subtropical de acordo com a classificação de Köppen-Geiger (Peel et al. 2007), foi quente e chuvoso durante o período de estudo.

Foram utilizados substratos artificiais para a colonização e desenvolvimento das algas perifíticas, o que oferece as seguintes vantagens: a) superfície uniforme; b) facilidade de determinação da área colonizada; c) possibilidade de definir o tempo de colonização e os estádios de sucessão (Moschini-Carlos 1999). Assim, optou-se por um substrato semelhante às macrófitas aquáticas submersas, enraizadas no sedimento, abundantes no ambiente, porém de difícil remoção das algas devido à grande quantidade de folhas frágeis. Cada substrato artificial foi lavado em água corrente; posteriormente, ramos foram presos a um peso por uma linha de náilon e a pedaços de isopor, que serviram como bóias. Estes substratos foram instalados a uma profundidade aproximada de $60 \mathrm{~cm}$, próximo à margem esquerda do rio, $\mathrm{e}$ coletados ao longo do estudo de modo aleatório. O perifíton foi removido dos substratos com auxílio de uma escova e jatos de água destilada, sendo depois transferido para frascos $(150 \mathrm{~mL})$, fixado e preservado com solução de lugol acético $0,5 \%$.

Os substratos artificiais empregados foram similares em forma e tamanho, possuindo área de $11,13 \mathrm{~cm}^{2}$. Para a estimativa da área do substrato artificial, uma planta de plástico foi cortada, todos os segmentos e o eixo principal foram digitalizados e as imagens foram transferidas para o programa Imagem-Pro Plus versão 4.5.0.29 (Media Cybernetics Inc. 2001).

Variáveis abióticas foram medidas no momento de coleta dos substratos artificiais: temperatura da água (oxímetro portátil marca YSI modelo 55), condutividade elétrica (condutivímetro portátil Digimed modelo DM2), $\mathrm{pH}$ (medidor de pH portátil Digimed modelo DM2), turbidez (turbidímetro portátil modelo Lamotte), vento (anemômetro). Amostras de 1 litro de água foram coletadas na sub-superfície da coluna de água para determinação dos seguintes nutrientes: nitrato $=\mathrm{NO}_{3}^{-}$(Mackereth et al. 1978), amônio $=\mathrm{NH}_{4}^{+}$(Solorzano 1969), nitrogênio total $=\mathrm{NT}$ (Valderrama 1981), fósforo total $=$ PT $($ Valderrama 1981), fósforo dissolvido total $=$ PDT (Valderrama 1981) e ortofosfato $=\mathrm{PO}_{4}^{-3}$ (Golterman et al. 1978).

A identificação e a quantificação das algas foram realizadas em microscópio invertido, marca Olympus CK-2, utilizando o método de Utermöhl (1958). Os indivíduos foram contados em campos aleatórios (Bicudo 1990), até atingir a estabilização da curva e até que as espécies abundantes contabilizassem um mínimo de 100 indivíduos. $\mathrm{O}$ cálculo quanto à eficiência de contagem de espécies e número de indivíduos de cada espécie variou de 90 a 97\%, seguindo o critério de Pappas \& Stoermer (1996). O enquadramento taxonômico adotado foi o proposto por Round $(1965,1971)$, seguindo-se recomendação de Bicudo \& Menezes (2006). Os valores da densidade foram convertidos por unidade de área do substrato $\left(\mathrm{cm}^{2}\right)$, através da equação adaptada por Ros (1979).

Para avaliar a estrutura da comunidade, foram utilizadas: a) densidade expressa em número de indivíduos por $\mathrm{cm}^{2}$; b) diversidade de espécies (índice de Shannon-Wiener) e equitabilidade (índice 
de Simpson), os quais levam em consideração o número de espécies (riqueza) e o número de indivíduos em cada espécie (abundância). Foram empregadas as seguintes categorias quanto à abundância relativa de cada táxon: dominante (D) $=$ acima de 10.000; abundante $(\mathrm{A})=5.000$ a 10.000 ; numeroso $(\mathrm{N})=2.000$ a $5.000 ; \operatorname{comum}(\mathrm{C})=500$ a 2.000; pouco frequente $(\mathrm{PF})=100$ a 500 ; raro $(\mathrm{R})$ $=10$ a 100 (Brown 1976).

A contribuição relativa das espécies mais abundantes (descritoras da comunidade) em cada estádio da sucessão foi definida de acordo com a soma dos valores da densidade das espécies que tiveram uma porcentagem acima de $2 \%$ da densidade total e que juntas atingissem mais de $50 \%$ da densidade total de cada estádio.

Foi construída uma matriz com as variáveis abióticas (13) e os tempos de sucessão (8) para a Análise de Componentes Principais (ACP), usando o programa PC-ORD, versão 5.0. A análise foi realizada após transformação das variáveis [(Xij$\mathrm{Xi} / \mathrm{Si}$ ]. Para a interpretação dos eixos, foram considerados os eixos com valores maiores que o modelo de Broken-Stick (Jackson 1993).

\section{Resultados}

Houve maior volume de precipitação no período compreendido entre o final de novembro (final da $1^{\mathrm{a}}$ semana de estudo) e início de dezembro ( $2^{\mathrm{a}}$ semana) (Fig. 2).

Os dois primeiros eixos da ACP explicaram $71,7 \%$ da variabilidade conjunta dos dados abióticos (Fig. 3, Tab. 1). No eixo 1 (49,2\%), tempo 0 e $6^{\circ}$ dia (fase inicial da sucessão) ficaram próximos no lado positivo, relacionando-se aos maiores valores de nitrato, $\mathrm{pH}$ e nitrogênio total, enquanto $15^{\circ}$ e $18^{\circ}$ dias, posicionados no lado negativo, foram relacionadas aos maiores valores de fósforo total, fósforo dissolvido total e turbidez. No eixo $2(22,6 \%), 9^{\circ}$ e $12^{\circ}$ dias ficaram próximos, em função dos maiores valores de precipitação, enquanto $3^{\circ}$ e $21^{\circ}$ dias se posicionaram mais distantes dos demais dias, devido aos maiores valores de temperatura da água, condutividade e oxigênio dissolvido (Tab. 1, Fig. 3).

A densidade total do ficoperifíton variou de 349 ( $3^{\circ}$ dia de sucessão) a $1.863 \times 10^{3}$ ind.cm ${ }^{-2}\left(15^{\circ}\right.$ dia, Fig. 4). A densidade do $15^{\circ}$ dia apresentou valor mais elevado que as densidades dos $3^{\circ}, 6^{\circ}, 9^{\circ}, 12^{\circ}$ e $21^{\circ}$ dias de colonização.

Ao analisar o número de indivíduos em cada classe, foi verificado o predomínio de

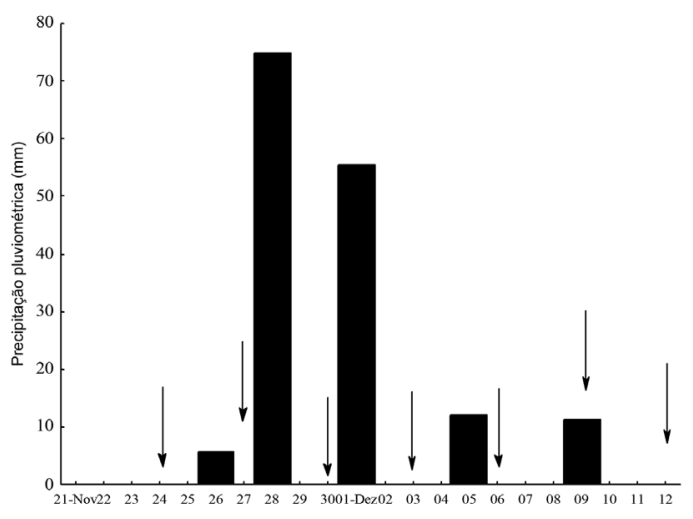

Figura 2 - Precipitação no rio do Corvo, reservatório de Rosana, Estado do Paraná, Brasil, no período de 21 de novembro a 12 de dezembro de 2003. Setas indicam quando as coletas do perifíton foram realizadas.

Figure 2 - Precipitation on Corvo stream, Rosana reservoir, Paraná state, Brazil, between 21 November and 12 December 2003. Arrows indicate when periphyton samples were taken.

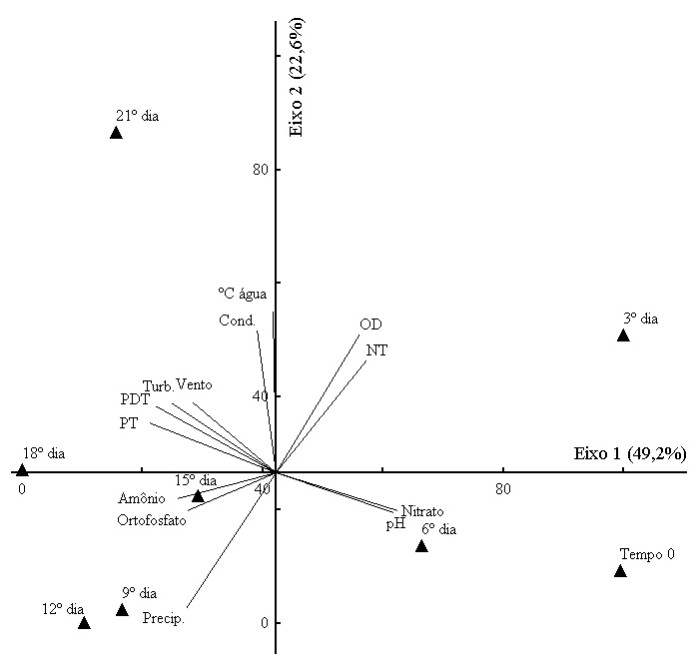

Figura 3 - Análise de componentes principais (ACP) com base em matriz de variáveis abióticas (13) e tempos de sucessão (8) em comunidade perifitica em substratos artificiais, submersos no rio do Corvo, analisada no período de 21 de novembro a 12 de dezembro de $2003 .{ }^{\circ} \mathrm{C}$ água $=$ temperatura da água, Cond. = condutividade elétrica, $\mathrm{NT}=$ nitrogênio total, $\mathrm{OD}=$ oxigênio dissolvido, $\mathrm{PDT}=$ fósforo total dissolvido, Precip. $=$ precipitação, $\mathrm{PT}=$ fósforo total, Turb. $=$ turbidez.

Figure 3 - Principal component analysis (PCA) based on abiotic variables (13) and succession times (8) of the periphytic algal community on artificial substrate when submerged in Corvo stream, analyzed from 12 November to 12 December 2003 . ${ }^{\circ} \mathrm{C}$ água = water temperature, Cond. = electrical conductivity, $\mathrm{NT}=$ total nitrogen, $\mathrm{OD}=$ dissolved oxygen, $\mathrm{PDT}=$ total dissolved phosphorus, Precip. $=$ precipitation, $\mathrm{PT}=$ total phosphorus, Turb. $=$ turbidity. 
Tabela 1- Correlação de Pearson entre as variáveis abióticas e os dois primeiros eixos da Análise de Componentes Principais.

Table 1 - Pearson Correlation of abiotic variables with the first two axes of Principal Components Analysis.

\begin{tabular}{lcc}
\hline Variáveis abióticas & Eixo 1 & Eixo 2 \\
\hline $\mathrm{pH}$ & 0.8667 & -0.1975 \\
Condutividade & -0.1375 & 0.7081 \\
Oxigênio dissolvido & 0.6152 & 0.6882 \\
Temperatura da água & -0.021 & 0.8057 \\
Turbidez & -0.771 & 0.3458 \\
Fósforo total & -0.9284 & 0.248 \\
Fósforo total dissolvido & -0.8865 & 0.3302 \\
Ortofosfato & -0.6494 & -0.1882 \\
Nitrogênio total & 0.6677 & 0.5589 \\
Nitrato & 0.8908 & -0.1881 \\
Ion amônio & -0.7244 & -0.1314 \\
Vento & -0.6176 & 0.3503 \\
Precipitação & -0.6592 & -0.675 \\
\% total da variância & 49.176 & 22.555 \\
\% acumulada da variância & 49.176 & 71.73 \\
\hline
\end{tabular}

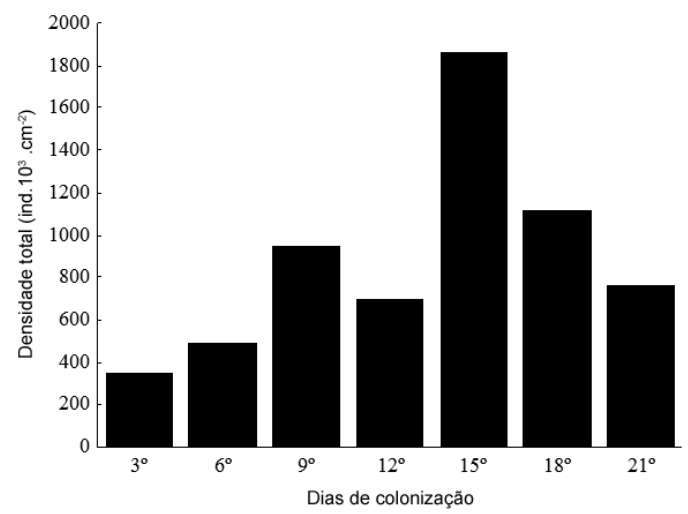

Figura 4 - Variação da densidade total de algas perifíticas, analisada em substratos artificiais submersos no rio do Corvo, reservatório de Rosana, Estado do Paraná, Brasil, no período de 21 de novembro a 11 de dezembro de 2003.

Figure 4 - Total density variation of periphytic algae on artificial substrate submerged on Corvo stream, Rosana reservoir, Paraná state, Brazil, analyzed in the period of 21 November to 12 December 2003. algas unicelulares da classe Bacillariophyceae (diatomáceas) (Apêndice I). De forma geral, as diatomáceas apresentaram densidade média elevada em comparação com as demais classes (Fig. 5). No $3^{0}$ dia, as diatomáceas atingiram $80,6 \%$ da densidade total. No $6^{\circ}$ dia, a representatividade das diatomáceas em termos de densidade total diminuiu para $73,9 \%$, variando entre $79 \%$ e $80 \%$ entre os $9^{0}$ e $15^{\circ}$ dias. No $18^{\circ}$ dia, a densidade das diatomáceas voltou a diminuir, passando a representar 72,4\% do total (Fig. 5).

As densidades de todas as demais classes de algas (19 a 27,6\% de táxons) foram mais elevadas nos $15^{\circ}$ e $18^{\circ}$ dias (Fig. 5). Cyanophyceae foi a segunda classe em densidade nos $12^{\circ}, 15^{\circ}, 18^{\circ}$ e $21^{\circ}$ dias. Zygnemaphyceae foi a segunda mais representativa nos $3^{\circ}, 6^{\circ}$ e $9^{\circ}$ dias sucessionais. A contribuição das Chlorophyceae aumentou no $6^{\circ}$ dia (terceira maior classe em densidade), apresentando maiores valores nos $15^{\circ}$ e $18^{\circ}$ dias. A participação das Xanthophyceae, também observadas no $18^{\circ}$ dia, foi representada por 4,42 x $10^{3}$ ind. $\mathrm{cm}^{-2}$. Oedogoniophyceae, representada pelos gêneros Oedogonium e Bulbochaete, foi a terceira classe com maior densidade no $21^{\circ}$ dia de sucessão (Fig. 5).

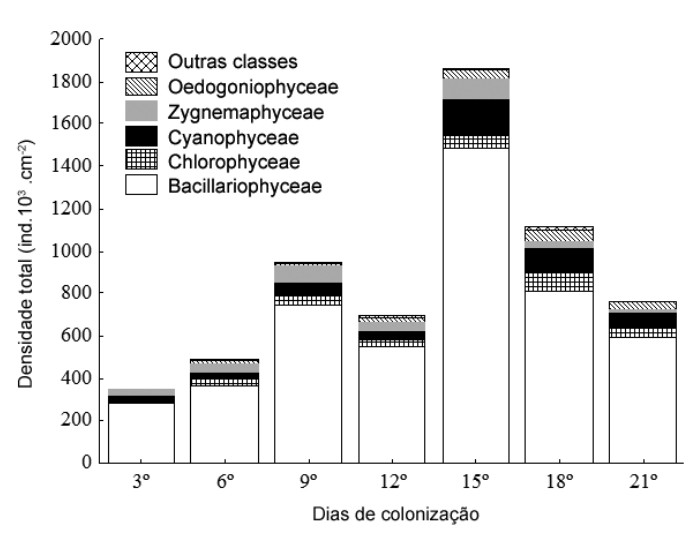

Figura 5 - Variação da densidade das classes de algas perifíticas em substratos artificiais submersos no rio do Corvo, reservatório de Rosana, Estado do Paraná, Brasil, analisada no período de 21 de novembro a 11 de dezembro de 2003. Chrysophyceae, Cryptophyceae, Euglenophyceae, Ulothricophyceae e Xanthophyceae foram agrupadas como outras classes.

Figure 5 - Density variation of periphytic algal classes on artificial substrate submerged on Corvo stream, Rosana reservoir, Paraná state, Brazil, analyzed in the period of 21 November to 12 December. Chrysophyceae, Cryptophyceae, Euglenophyceae, Ulothricophyceae and Xanthophyceae were grouped into other classes. 
O número de táxons representados nas categorias numeroso, abundante e dominante pertenceu às diatomáceas (31, 57 e 75, respectivamente), o que representou de 77,8 a $100 \%$. Por outro lado, a maioria dos incluídos nas categorias comum, pouco frequente e raro pertenceu a Zygnemaphyceae $(10,34$ e 36, respectivamente), representando cerca de 42,8 a 56,2\% dos taxons (Apêndice 1).

As espécies raras ocorreram no início do processo de colonização, sobretudo no $6^{\circ}$ dia $(26$ táxons ao todo, Fig. 6), sendo que Zygnemaphyceae teve maior número de espécies (53\% no início da sucessão e $36 \%$ no $9^{\circ}$ dia). Os táxons dominantes, comuns e pouco frequentes apresentaram maior número de espécies no $15^{\circ}$ dia sucessional (Fig. 6). Eunotia lineolata Hust., E. flexuosa Bréb. e Oedogonium spp. aumentaram a ocorrência de pouco frequentes e comuns nos $3^{\circ}$ e $6^{\circ}$ dias para numerosos a abundantes nos estádios seguintes. Pseudanabaena catenata Laut. e Stigeoclonium spp. passaram de pouco frequentes no início, para numerosos no final do período de estudo.

Fragilaria capucina Desm. foi a única espécie dominante durante todo o período (Fig. 7). Os táxons mais abundantes no $3^{\circ}$ dia sucessional contribuíram com $72 \%$ da comunidade de algas perifíticas, incluindo Gomphonema parvulum (Kütz.) Kütz., Ulnaria ulna (Nitz.) Com., Achnanthidium minutissimum (Kütz.) Czar., P. catenata, Eunotia sudetica O. Müll., Encyonema lunatum (Smith) Van Heurck e $F$. capucina, enquanto no $6^{\circ}$ dia a contribuição foi de $68 \%$. Porém, U. ulna e E. lunatum tiveram abundância menor e foram substituídas por Cymbella sp. e Encyonema minutum (Hil.) Man. (Fig. 7a e 7b). No $9^{\circ}$ dia, estes dois últimos táxons, e E. lunatum, Komvophoron crassum (Voz.) Anag. et Kom., A. minutissimum, $G$. parvulum e $F$. capucina contribuíram com $72 \%$ (Fig. 7c). No $12^{\circ}$ dia, E. camelus Ehr., E. sudetica, Oedogonium sp. 3, P. catenata e E. mesianum (Chol.) Mann substituiram Cymbella sp. e $K$. crassum quanto à maior abundância (Fig. 7d). Komvophoron minutum (Skuja) Anag. \& Kom. passou a integrar o grupo de espécies mais abundantes e, com as demais, contribuíram com 73,5\% da comunidade no $15^{\circ}$ dia (Fig. 7e). Oedogonium sp. 2, Chroococcus minimus (Keis.) Lemm., Gomphonema gracile Ehr., Achnanthidium exiguum (Grun.) Czar., E. sudetica, Oedogonium sp. 4, junto com os outros táxons, contribuíram com mais de $70 \%$ da comunidade no $18^{\circ}$ dia (Fig. $7 f$ ). Apesar do último dia $\left(21^{\circ} \mathrm{dia}\right)$ apresentar táxons com maior contribuição na comunidade (Fig. 7g), estes táxons estiveram mais próximos em relação à densidade, o que diferiu dos $15^{\circ}, 9^{\circ}, 6^{\circ}$ e $3^{\circ}$ dias, nos quais $F$. capucina destacou-se dos demais.

Dentre os 35 táxons do modelo de matriz conceitual para ambientes lóticos temperados, cinco diatomáceas foram aqui abundantes durante todo o período: A. minutissimum, E. minutum, G. parvulum, F. capucina e U. ulna (Fig. 7), juntamente com Cymbella affinis Kütz., abundantes no $15^{\circ}$ dia, estão entre as $r$-estrategistas. Entre os nove táxons C-S-estrategistas descritos por Biggs et al. (1998), seis (Mougeotia, Navicula cryptocephala Kütz., Oedogonium, Phormidium, Spirogyra e Stigeoclonium) foram aqui descritos como numerosos, principalmente a partir do $12^{\circ}$ dia sucessional, ou seja, a partir dos estádios intermediários até o período final, em especial Oedogonium e Stigeoclonium.

A riqueza de espécies (S) (Apêndice 1) e a diversidade de Shannon-Wiener $(\mathrm{H})$ apresentaram tendências muito similares (Fig. 8). No entanto, a riqueza de espécies e a diversidade de ShannonWiener atingiram valores máximos nos $15^{\circ}$ e $18^{\circ}$ dias de sucessão, respectivamente. A equitabilidade e o índice de dominância na comunidade de algas perifíticas apresentaram maiores valores $(0,66$ e 0,86 , respectivamente) nas fases finais da sucessão, quando a riqueza de espécies foi mais baixa (Fig. 8).

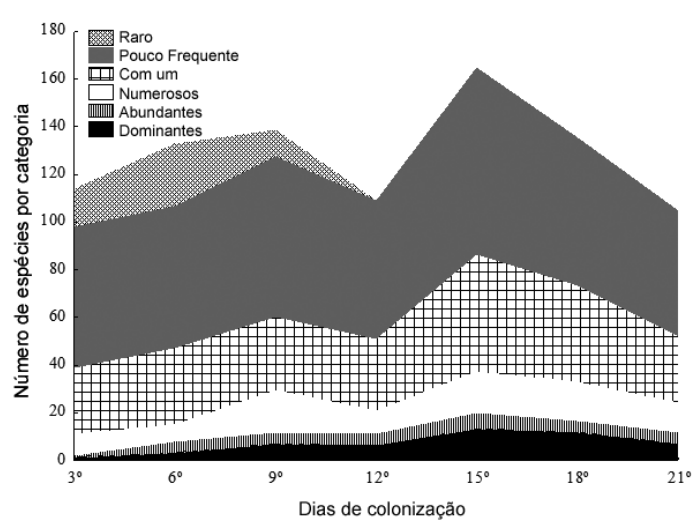

Figura 6 - Número de espécies, por classes de abundância, de algas perifíticas em substratos artificiais, submersos no rio do Corvo, reservatório de Rosana, Estado do Paraná, Brasil, analisadas no período de 21 de novembro a 12 de dezembro de 2003.

Figure 6 - Number of species of periphytic algae on artificial substrate submerged on Corvo stream, Rosana reservoir, Paraná state, Brazil, analyzed in the period of 21 November to 12 December 2003, represented by different abundance classes. 

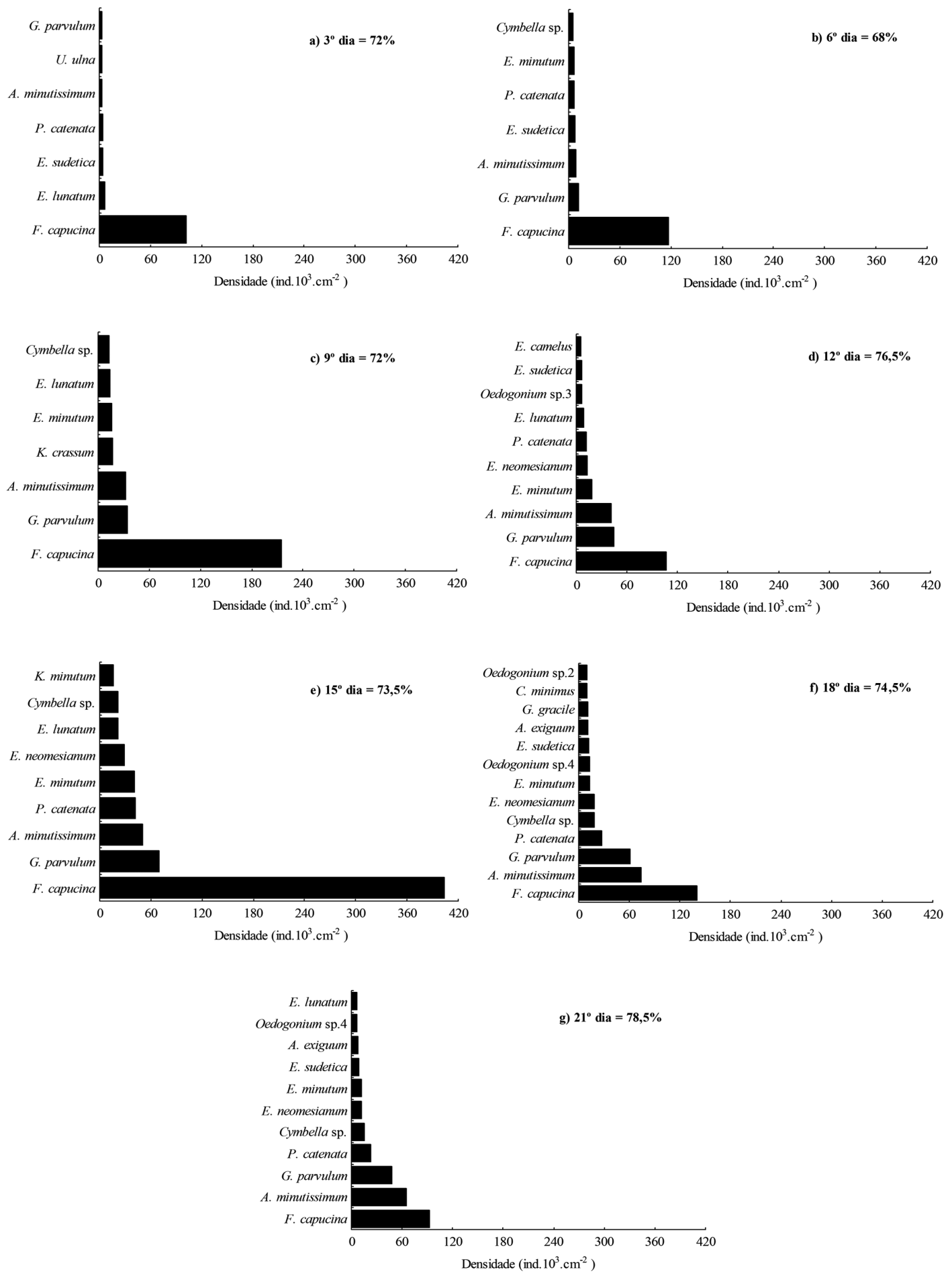

Figura 7 - Espécies mais abundantes (descritoras) de algas perifíticas em substratos artificiais, submersos no rio do Corvo, reservatório de Rosana, Estado do Paraná, Brasil, em cada tempo de sucessão, analisada no período de 21 de novembro a 12 de dezembro de 2003. Os valores em percentual (soma das densidades das espécies) estão indicados nas letras de a-g. Figure 7 - Most abundant species (descriptor species) in the periphytic algal community on artificial substrates when submerged in the Corvo stream, Rosana reservoir, Paraná state, Brazil, in each succession time, analyzed in the period of 21 November to 12 December 2003. The values in percentage (sum of species densities) are indicated in the letters a-g. 


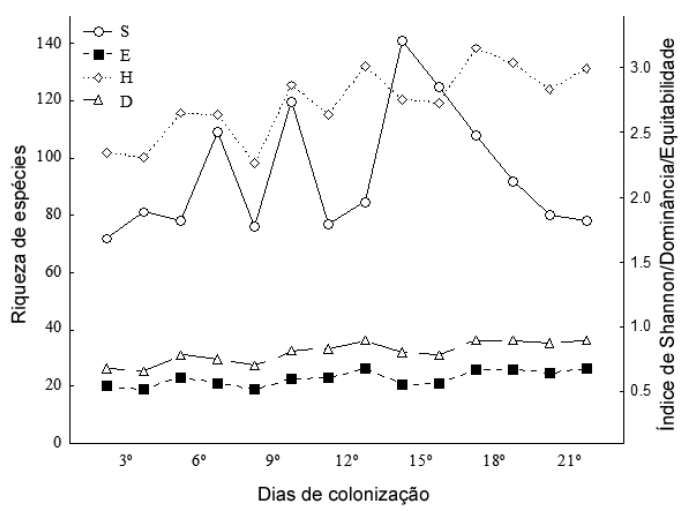

Figura 8 - Riqueza de espécies (S), diversidade de Shannon-Wiener $(\mathrm{H})$, índice de dominância (D) e equitabilidade (E) em comunidades de algas perifíticas, em substratos artificiais submersos no rio do Corvo, reservatório de Rosana, Estado do Paraná, Brasil, analisadas no período de 21 de novembro a 12 de dezembro de 2003. Figure 8 - Species richness (S), Shannon-Wiener diversity (H), dominance (D) and evenness indexes (E) in periphytic algal community on artificial substrate when submerged in Corvo stream, Rosana reservoir, Paraná state, Brazil, analyzed in the period of 21 November to 12 December 2003.

\section{Discussão}

A sucessão das algas perifíticas no rio do Corvo foi caracterizada principalmente pela maior abundância de espécies pequenas de diatomáceas, consideradas colonizadores e de crescimento rápido, durante todo o período de estudo; e de cianobactérias e algas verdes filamentosas, de crescimento mais lento, mais abundantes nos estádios tardios. As primeiras são consideradas, de acordo com Biggs et al. (1998), como $r$-estrategistas, enquanto as últimas estão entre as $\mathrm{C}-\mathrm{S}$ estrategistas. As diatomáceas menores foram os colonizadores iniciais em substrato artificial em ambiente lótico subtropical (Rodrigues \& Bicudo 2004), como também documentado para regiões temperadas (Hoagland et al. 1982, 1986; Biggs 1996).

O predomínio das diatomáceas pode estar relacionado ao fato de muitas espécies terem distribuição mais ampla em relação às variações ambientais, apresentarem estratégias adaptativas com alta resistência a distúrbios físicos, grande capacidade de adesão a substratos (formas adnatas presas pelo canal da rafe), altas taxas de migração e tamanho relativamente pequeno (Biggs et al. 1998; Stoermer \& Julius 2003). Muitas espécies de Cymbella, Encyonema, Gomphonema, Synedra e Eunotia possuem mecanismos de adaptação aos diversos substratos, como pedúnculos de fixação ao substrato e poros mucilaginosos que facilitam a adesão ao substrato (Hoagland et al. 1982; Rodrigues \& Bicudo 2004).

Padrões nas características de crescimento populacional em diatomáceas de diferentes espécies indicam diferentes estratégias ecológicas. Estas estratégias variam de espécies oportunistas, que se reproduzem rapidamente e dispersam quando o fornecimento de nutrientes se torna limitante, à espécies competitivas, com crescimento mais lento, que utilizam os recursos de forma mais eficiente (Stevenson et al. 1991). Estas estratégias vão se alterando, na habilidade de proliferação em espaços recentemente vazios (Peterson 1996; Biggs et al. 1998), na exploração de fontes de nutrientes inorgânicos localizadas e/ou passageiras, na resistência às ondas e correntes (Biggs \& Thomsen 1995). Tais habilidades para proliferar e resistir aos distúrbios revelam a grande plasticidade das espécies em se estabelecer e persistir em diferentes condições ambientais (Stevenson \& Peterson 1989; Stevenson et al. 1991; Biggs \& Thomsen 1995; Peterson 1996).

Este fato pode explicar a presença de Achnanthidium minutissimum, Encyonema mesianum, E. minutum, Eunotia sudetica, Fragilaria capucina e Gomphonema parvulum, espécies colonizadoras iniciais e que se mantiveram abundantes até o final do estudo. Tais espécies, junto com Cymbella affinis, são citadas por Biggs et al. (1998) como pertencentes a um grupo particular (seleção R) de espécies pequenas, colonizadoras, com crescimento rápido e baixa biomassa, encontradas em ambientes perturbados estáveis, com fornecimento de recursos de moderado a alto (mesotrófico). Por sua vez, táxons arrafides, como F. capucina e Ulnaria ulna, são colonizadores mais ativos que monorrafides e birrafides, uma vez que formam colônias que se aderem apicalmente ao substrato, projetando-se verticalmente (Stevenson \& Peterson 1989).

Dentre as algas verdes filamentosas citadas, no modelo de matriz conceitual de Biggs et al. (1998), Oedogonium, Spirogyra e Stigeoclonium, classificados como seleção $\mathrm{C}$-S, são mais competitivos em níveis intermediários de nutrientes em habitats estáveis, porém com filamentos mais vulneráveis ao arraste. Possivelmente, a presença de células basais, com estruturas para fixação ao 
substrato, tenha favorecido a permanência destes representantes nos estádios finais do estudo. Oedogonium pode ser encontrado aderido a diversos tipos de substratos (Lee 2008), e estar dominante em ambientes com disponibilidade de nutrientes e correnteza com baixa velocidade ou inexistente (Biggs 1996; Biggs et al. 1998). As formas de vida filamentosas dividiram a representatividade junto com formas unicelulares ao longo do processo sucessional, principalmente em níveis de distúrbios mais moderados (Rodrigues $\&$ Bicudo 2004).

Em determinados ambientes, a maior quantidade de substratos disponíveis para colonização do perifíton pode proporcionar maior heterogeneidade na comunidade e, assim, aumentar a diversidade de espécies (Stevenson 1997). Com isso, quanto mais diversas as condições do ambiente, maior poderá ser o número de espécies (Lampert \& Sommer 1997). No rio do Corvo, antes que a comunidade de algas perifíticas atingisse o pico de densidade, as oscilações registradas revelaram a capacidade de uma nova comunidade estabelecer-se ou recuperar-se, apesar das alterações/perturbações no ambiente. A relação do aumento da diversidade de espécies nas comunidades de algas perifíticas, com aumento da resiliência em ambientes mais heterogêneos, seria devido ao uso mais eficiente de uma quantidade maior de recursos disponíveis (Stevenson 1997; Lampert \& Sommer 1997). Os valores da diversidade de Shannon-Wiener deste estudo foram superiores $(2,2$ a 3,1$)$ aos encontrados por Lam \& Lei (1999) para ecossistema lótico tropical (1,5 a 2,3 ind.bits), com maiores índices de diversidade e riqueza registrados no $15^{\circ}$ dia de sucessão.

Neste estudo de curta duração, sugere-se que a concentração de nutrientes, o pH e a turbidez (como evidenciado na ACP), foram os principais fatores controladores do processo de colonização da comunidade perifítica, sendo ainda influenciada pela precipitação pluviométrica. É esperado que, durante o curso direcional da colonização, ocorram mudanças na composição de espécies, aumento da diversidade e substituição de espécies estrategistas $r$ em grande parte por estrategistas em $K$ (Odum 1988). Neste sentido, os resultados mostraram que as diatomáceas foram o grupo dominante, sendo $r$-estrategistas e os principais colonizadores, além de determinarem a diversidade durante todo o período. Desta forma, os resultados deste estudo confirmam a hipótese levantada no início.

\section{Agradecimentos}

Agradecemos ao Nupélia e PEA, pelo apoio logístico na execução do projeto e suporte técnico-científico. A CAPES, pela concessão da bolsa de doutorado. Ao Conselho Nacional de Desenvolvimento Científico e Tecnológico, pela bolsa de produtividade da segunda autora. Esta pesquisa está inserida no Projeto PRONEX "Produtividade em Reservatório: relações com o estado trófico e predação", desenvolvido pelo Nupélia.

\section{Referências}

Bicudo, C.E.M. \& Menezes, M. 2006. Gêneros de algas de águas continentais do Brasil. Rima, São Carlos. 512p.

Bicudo, D.C. 1990. Considerações sobre metodologias de contagem de algas do perifíton. Acta Limnologica Brasiliensis 3: 459-475.

Biggs, B.J.F. 1996. Patterns in benthic algal of streams. In: Stevenson, R.J.; Bothwell, M.L. \& Lowe, R.L. (eds). Algal ecology: freshwater benthic ecosystems. Academic Press, New York. Pp. 31-56.

Biggs, B.J.F.; Stevenson, R.J. \& Lowe, R.L. 1998. A habitat matrix conceptual model for stream periphyton. Archiv für Hydrobiologie 143: 21-56.

Biggs, B.J.F. \& Thomsen, H.A. 1995. Disturbance of stream periphyton by perturbations in shear stress: time to structural failure and differences in community resistence. Journal of Phycology 31: 233-241.

Borges, F.R. \& Necchi Jr., O. 2008. Short-term successional dynamics of a macroalgal community in a stream from northwestern São Paulo State, Brazil. Acta Botanica Brasilica 22: 453-463.

Branco, C.C.Z.; Branco, L.H.Z.; Moura, M.O. \& Bertusso F.R. 2005. The succession dynamics of a macroalga community after a flood disturbance in a tropical stream from São Paulo State, southeastern Brazil. Revista Brasileira de Botânica 28: 267-275.

Brown, H.D. 1976. A comparison of the attached algal communities of a natural and an artificial substrate. Journal of Phycology 12: 301-306.

Cetto, J.M.; Leandrini, J.A.; Felisberto, S.A. \& Rodrigues, L. 2004. Comunidade de algas perifíticas no reservatório de Irai, Estado do Paraná, Brasil. Acta Scientiarum, Biological Sciences 26: 1-7.

Felisberto, S.A. \& Rodrigues, L. 2005. Comunidade de algas perifíticas em reservatórios de diferentes latitudes. In: Rodrigues, L.; Thomaz, S.M.; Agostinho, A.A. \& Gomes, L.C. (eds). Biocenoses em reservatórios: padrões espaciais e temporais. Rima, São Carlos. Pp. 97-114.

Felisberto, S.A. \& Rodrigues, L. 2010. Periphytic algal community in artificial and natural substratum in a tributary of the Rosana reservoir (Corvo Stream, Paraná State, Brazil). Acta Scientiarum, Biological Sciences 32: 373-385. 
Golterman, H.L.; Clymo, R.S. \& Ohmstad, M.A.M. 1978. Methods for physical and chemical analysis of freshwaters. Blackwell Scientific, Oxford. 214p.

Hoagland, K.D.; Roemer, S.C. \& Rosowki, J.R. 1982. Colonization and community structure of two periphyton assemblages, with emphasis on the diatoms Bacillariophyceae. American Journal of Botany 69: 188-213.

Hoagland, K.D.; Zlotski, A. \& Peterson, C.G. 1986. The source of algal colonizer on rock substrates in a freshwater impoudment. In: Evans, L.V. \& Hoagland, K.D. (eds). Algal biofouling. Elsevier Science, Amsterdan. Pp. 21-39.

Jackson, D.A. 1993. Stopping rules in principal components analysis: a comparison of heuristical and statistical approaches. Ecology 74: 22042214.

Lam, P.K.S. \& Lei, A. 1999. Colonization of periphytic algae on artificial substrates in a tropical stream. Diatom Research 14: 307-322.

Lampert, W. \& Sommer, U. 1997. Limnoecology: the ecology of lakes and streams. Oxford University Press, New York. 382p.

Lee, R.E. 2008. Phycology. 2a ed. Cambridge University Press, Cambridge. 644p.

Mackereth, F.J.H.; Heron, J. \& Talling, J.F. 1978. Water analysis: some revised methods for limnologists. Titus Wilson \& Sons Ltd, Kendall. (Freshwater Biological Association Scientific Publication $\mathrm{n}^{\mathrm{o}}$ 36). 117p.

McCormick, P.V. \& Stevenson, R.J. 1991. Mechanisms of benthic algal succession in lotic environments. Ecology 72: 1835-1848.

Media Cybernetics. 2001. Imagem-Pro Plus. Versão 4.5.0.29 for Windows. Proven Solution, Inc., Silver Spring.

Moschini-Carlos, V. 1999. Importância, estrutura e dinâmica da comunidade perifítica nos ecossistemas aquáticos continentais. In: Pompêo, M.L.M. (ed.). Perspectivas da limnologia no Brasil. Gráfica e Editora União, São Luís. Pp. $1-11$.

Odum, E.P. 1969. The strategy of ecosystem development. Science 164: 262-270.

Odum, E.P. 1988. Ecologia. Ed. Guanabara, Rio de Janeiro. 434p.

Pappas, J.L. \& Stoermer, E.F. 1996. Quantitative method for determining a representative algal sample count. Journal of Phycology 32: 693-696.

Peel, M.C.; Finlayson, B.L. \& McMahon, T.A. 2007. Updated world map of the Köppen-Geiger climate classification. Hydrology and Earth System Sciences 11: 1633-1644.

Peterson, C.G. 1996. Mechanisms of lotic microalgal colonization following space-clearing disturbances acting at different spatial scales. Oikos 77: 417-435.

Peterson, C.G. \& Stevenson, R.J. 1992. Resistance and resilience of lotic algal communities: importance of disturbance timing and current. Ecology 73:1445-1461.

Rodrigues, L. \& Bicudo, D.C. 2001. Similarity among periphyton algal communities in a lentic-lotic gradient of the upper Paraná river floodplain, Brazil. Revista Brasileira de Botânica 24: 235-248.

Rodrigues, L. \& Bicudo, D.C. 2004. Periphytic algae. In: Thomaz, S.M.; Agostinho, A.A. \& Hahn, N.S. (eds). The upper Paraná River and its floodplain: physical aspects, ecology and conservation. Backhuys Publishers, Leiden. Pp. 125-143.

Ros, J. 1979. Prácticas de Ecologia. Ed. Omega, Barcelona. 181p.

Round, F.E. 1965. The biology of the algae. Edward Arnold Publs. Ltd, London. 269p.

Round, F.E. 1971. The taxonomy of the Chlorophyta, 2. British Phycological Journal 6: 235-264.

Solorzano, L. 1969. Determination of ammonia in natural waters by the phenolhypochlorite method. Limnology and Oceanography 14: 799-801.

Steinman, A.D.; Mulholland, P.J.; Palumbo, A.V.; Flum, T.F. \& DeAngelis, D.L. 1991. Resilience of lotic ecosystems to a light-elimination disturbance. Ecology 72: 1299-1313.

Stevenson, R.J. 1996. An introduction to algal ecology in freshwater benthic habitats. In: Stevenson, R.J.; Bothwell, M.L. \& Lowe, R.L. (eds). Algal ecology: freshwater benthic ecosystems. Academic Press, San Diego. Pp. 3-30.

Stevenson, R.J. 1997. Scale-dependent determinants and consequences of benthic algal heterogeneity. Journal of North American Benthological Society 16: 248-262.

Stevenson, R.J. \& Peterson, C.G. 1989. Variation in benthic diatom (Bacillariophyceae) immigration with habitat characteristics and cell morphology. Journal of Phycology 25: 120-129.

Stevenson, R.J.; Peterson, C.G.; Kirschtel, D.B.; King, C.C. \& Tuchman, N.C. 1991. Densitydependent growth, ecological strategies, and effects of nutrients and shading on benthic diatom succession in streams. Journal of Phycology 27: 59-69.

Stoermer, E.F. \& Julius, M.L. 2003. Centric diatoms. In: Wehr, J.D. \& Sheath, R.G. (eds). Freshwater algae of North America: ecology and classification. Academic Press, San Diego. Pp. 559-594. 
Townsend, C.R.; Begon, M. \& Harper, J.P. 2010. Fundamentos em ecologia. $3^{\text {a }}$. ed. Ed. Artmed, Porto Alegre. 576p.

Utermöhl, H. 1958. Zur Vervollkommung der quantitativen phytoplankton-methodik. Mitteilungen. Internationale Vereiningung fuer Theoretische und Angewandte Limnologie 9: $1-38$.
Valderrama, J.C. 1981. The simultaneous analysis of total nitrogen and total phosphorus in natural waters. Marine Chemistry 10: 109-122.

Vercellino, I.S. \& Bicudo, D.C. 2006. Sucessão da comunidade de algas perifíticas em reservatório oligotrófico tropical (São Paulo, Brasil): comparação entre período seco e chuvoso. Revista Brasileira de Botânica 29: 363-377. 


\title{
Dinâmica sucessional de comunidade de algas perifíticas em um ecossistema lótico subtropical
}

Successional dynamic of the periphytic algal community in ecossistem lotic subtropical

\author{
Sirlene Aparecida Felisberto \& Liliana Rodrigues
}

Apêndice 1 - Lista de táxons da comunidade de algas perifíticas analisados para cada dia de colonização, no rio do Corvo, (reservatório de Rosana). Abundância relativa: $\mathrm{D}=$ espécies dominantes; $\mathrm{A}=$ abundantes; $\mathrm{N}=$ numerosos; $\mathrm{C}=$ comum; $\mathrm{PF}=$ pouco frequente; $\mathrm{R}=$ raro.

Appendix 1 - List of taxa in the periphytic algal community analyzed for each day of colonization, in the Corvo Stream (tributary of Rosana reservoir). Relative abundance: $\mathrm{D}=$ dominant species; $\mathrm{A}=$ abundant; $\mathrm{N}=$ many; $\mathrm{C}=$ common; $\mathrm{PF}=$ infrequent; $\mathrm{R}=$ rare.

\begin{tabular}{llllllll}
\hline DIAS DE COLONIZAÇÃO & $3^{\circ}$ & $6^{\circ}$ & $9^{\circ}$ & $12^{\circ}$ & $15^{\circ}$ & $18^{\circ}$ & $21^{\circ}$ \\
\hline
\end{tabular}

Bacillariophyceae

Achnanthidium exiguum (Grun.) Czarn.

Achnanthidium minutissimum (Kütz.) Czarn.

Amphora sp.

Aulacoseira granulata (Ehr.) Sim.

Cyclotella cf. stelligera (Cleve \& Grun.) V. Heur.

$\begin{array}{ccccccc}\text { PF } & \text { C } & \text { C } & \text { C } & \text { N } & \text { D } & \text { A } \\ \text { N } & \text { A } & \text { D } & \text { D } & \text { D } & \text { D } & \text { D } \\ - & - & - & - & - & \text { PF } & - \\ - & \text { PF } & \text { PF } & - & - & - & -\end{array}$

Cymbella affinis Kütz.

PF

Cymbella cuspidata Kütz.

Cymbella cf. mycrocephala Grun.

Cymbopleura naviculiformis (Auer.) Krammer

Diatoma sp.

Diploneis cf. subovalis Cleve

$\mathrm{PF} \quad-\quad-\quad \mathrm{N} \quad \mathrm{A} \quad-\quad \mathrm{PF}$

Encyonema lunatum (Smith) V. Heur.

Encyonema mesianum (Chol.) Mann

Encyonema minutum (Hil.) Mann

Encyonema cf. perpusillum Cleve \& Mann

Encyonema silesiacum (Bleis.) Mann

Encyonema sp. 1

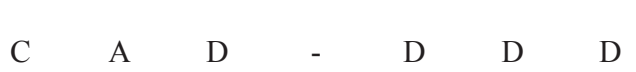

Encyonema sp. 2

$\begin{array}{ccccccc}\text { PF } & - & \text { C } & - & \text { PF } & \text { PF } & - \\ - & \text { PF } & - & - & \text { N } & - & \text { C } \\ - & \text { PF } & - & - & - & - & \text { PF } \\ \text { A } & \text { N } & \text { D } & \text { A } & \text { D } & \text { A } & \text { A } \\ \text { PF } & \text { N } & \text { A } & \text { D } & \text { D } & \text { D } & \text { D } \\ \text { N } & \text { A } & \text { D } & \text { D } & \text { D } & \text { D } & \text { D } \\ \text { PF } & - & - & - & - & - & - \\ - & - & \text { PF } & \text { N } & \text { D } & \text { A } & \text { N } \\ \text { C } & \text { A } & \text { D } & - & \text { D } & \text { D } & \text { D } \\ - & - & - & - & - & \text { PF } & - \\ \text { C } & \text { PF } & - & - & \text { PF } & \text { PF } & \text { PF }\end{array}$

Eunotia bilunaris Ehr. 


\section{DIAS DE COLONIZAÇÃO}

Eunotia camelus Ehr.

Eunotia flexuosa Bréb.

Eunotia formica Ehr.

Eunotia indica Grun.

Eunotia lineolata Hust.

Eunotia cf. minor (Kütz) Grun.

Eunotia cf. monodon Ehr.

Eunotia cf. pectinalis (Dillw.) Rab.

Eunotia pectinalis (Dillw.) Rab. var. ventricosa Grun.

Eunotia cf. sudetica O. Müll.

Eunotia zygodon Ehr.

Fragilaria acus var. angustissima

Fragilaria capucina Desm.

Fragilaria capucina Desm. forma teratologica

Fragilaria delicatissima (Smith) Lang.-Bert.

Frustulia rhomboides (Ehr.) De Toni

Frustulia cf. vitrea Oestrup

Gomphonema cf. brasiliense Grun.

Gomphonema complexo gracile Ehr.

Gomphonema parvulum Kütz.

Gomphonema cf. truncatum Ehr.

Gomphonema sp.

Hantzschia amphioxys (Ehr.) Grun.

Navicula cf. aikenensis Patr.

Navicula complexo "crypto"

Nitzschia cf. amphibia Grun.

Nitzschia cf. linearis Grun.

Nitzschia cf. palea (Kütz.) Smith

Nitzschia sp.

Pinnularia cf. braunii Grun.

\begin{tabular}{|c|c|c|c|c|c|c|}
\hline $3^{0}$ & $6^{0}$ & $9^{\circ}$ & $12^{\circ}$ & $15^{\circ}$ & $18^{\circ}$ & $21^{\circ}$ \\
\hline- & - & $\mathrm{PF}$ & A & $\mathrm{N}$ & $\mathrm{C}$ & $\mathrm{C}$ \\
\hline $\mathrm{C}$ & $\mathrm{PF}$ & $\mathrm{N}$ & $\mathrm{C}$ & $\mathrm{C}$ & $\mathrm{C}$ & $\mathrm{N}$ \\
\hline- & $\mathrm{C}$ & - & - & - & - & - \\
\hline $\mathrm{PF}$ & - & - & - & $\mathrm{PF}$ & $\mathrm{C}$ & - \\
\hline- & - & $\mathrm{C}$ & $\mathrm{N}$ & $\mathrm{D}$ & $\mathrm{N}$ & $\mathrm{N}$ \\
\hline $\mathrm{C}$ & $\mathrm{PF}$ & $\mathrm{N}$ & $\mathrm{PF}$ & - & $\mathrm{C}$ & $\mathrm{C}$ \\
\hline - & - & - & $\mathrm{PF}$ & $\mathrm{PF}$ & - & - \\
\hline- & $\mathrm{N}$ & $\mathrm{N}$ & $\mathrm{C}$ & $\mathrm{PF}$ & $\mathrm{N}$ & $\mathrm{C}$ \\
\hline $\mathrm{C}$ & - & $\mathrm{C}$ & - & - & - & - \\
\hline $\mathrm{N}$ & A & A & A & A & $\mathrm{D}$ & A \\
\hline- & - & - & - & - & $\mathrm{PF}$ & - \\
\hline- & - & - & - & - & - & $\mathrm{PF}$ \\
\hline $\mathrm{D}$ & $\mathrm{D}$ & $\mathrm{D}$ & $\mathrm{D}$ & $\mathrm{D}$ & $\mathrm{D}$ & $\mathrm{D}$ \\
\hline $\mathrm{N}$ & $\mathrm{C}$ & $\mathrm{N}$ & $\mathrm{C}$ & $\mathrm{N}$ & $\mathrm{N}$ & $\mathrm{C}$ \\
\hline $\mathrm{C}$ & $\mathrm{R}$ & $\mathrm{N}$ & $\mathrm{N}$ & $\mathrm{D}$ & $\mathrm{N}$ & $\mathrm{N}$ \\
\hline $\mathrm{PF}$ & - & $\mathrm{C}$ & $\mathrm{PF}$ & $\mathrm{PF}$ & $\mathrm{PF}$ & $\mathrm{PF}$ \\
\hline- & - & - & - & $\mathrm{PF}$ & - & - \\
\hline $\mathrm{PF}$ & - & - & - & - & - & - \\
\hline $\mathrm{N}$ & $\mathrm{C}$ & $\mathrm{N}$ & $\mathrm{N}$ & $\mathrm{N}$ & $\mathrm{D}$ & $\mathrm{N}$ \\
\hline $\mathrm{N}$ & $\mathrm{D}$ & $\mathrm{D}$ & $\mathrm{D}$ & $\mathrm{D}$ & $\mathrm{D}$ & $\mathrm{D}$ \\
\hline- & - & - & - & $\mathrm{PF}$ & - & - \\
\hline $\mathrm{C}$ & $\mathrm{C}$ & $\mathrm{C}$ & - & $\mathrm{C}$ & - & - \\
\hline- & - & - & - & - & $\mathrm{C}$ & $\mathrm{PF}$ \\
\hline- & - & - & - & $\mathrm{C}$ & $\mathrm{PF}$ & $\mathrm{PF}$ \\
\hline $\mathrm{C}$ & $\mathrm{N}$ & $\mathrm{N}$ & $\mathrm{N}$ & A & A & $\mathrm{C}$ \\
\hline- & - & - & $\mathrm{PF}$ & - & $\mathrm{C}$ & $\mathrm{PF}$ \\
\hline- & - & - & $\mathrm{PF}$ & $\mathrm{PF}$ & $\mathrm{PF}$ & - \\
\hline $\mathrm{C}$ & $\mathrm{C}$ & $\mathrm{N}$ & $\mathrm{C}$ & A & $\mathrm{C}$ & $\mathrm{PF}$ \\
\hline- & - & $\mathrm{PF}$ & - & - & - & - \\
\hline $\mathrm{R}$ & $\mathrm{PF}$ & - & - & - & - & - \\
\hline
\end{tabular}




\section{DIAS DE COLONIZAÇÃO}

Pinnularia $\mathrm{cf}$. divergens Smith

Pinnularia cf. interrupta Smith

Pinnularia maior (Kütz.) Rab.

Pinnularia cf. microstauron (Ehr.) Cleve

Pinnularia sp.

Planothidium lanceolatum (Bréb.) Round \& Bukh

Sellaphora pupulla Kütz.

Stauroneis phoenicenteron (Nitz.) Ehr.

Stenopterobia delicatissima (Lew.) Bréb. ex V. Heur.

Stenopterobia intermedia (Lew.) V. Heur.

Surirella cf. linearis Smith

Surirella sp.

Ulnaria ulna (Nitz.) Ehr.

Penales não identificada 1

Penalles não identificada 2

Chlorophyceae

Ankistrodesmus fusiformis Corda

Aphanochaete repens Braun

Bicuspidela cf. incus Pasch.

Botryococcus sp.

Chaetosphaeridium globosum (Nord.) Kleb.

Chaetosphaeridium sp.

Characiellopsis skujae (Fott) Kom.

Characium cf. ambiguum Herm.

Characium cf. cucurbitinum (Biss.) Teil.

Characium ornithocephalum (Braun)

Characium ornithocephalum (Braun) var. pringsheimii (Braun) Kom.

Chlamydomonas cf. globosa Snow

Closteriopsis acicularis (Smith) Belc. \& Swale

Coelastrum indicum Turner

$\begin{array}{lllllll}3^{\circ} & 6^{\circ} & 9^{\circ} & 12^{\circ} & 15^{\circ} & 18^{\circ} & 21^{\circ}\end{array}$

\begin{tabular}{|c|c|c|c|c|c|c|}
\hline- & - & - & - & - & PF & PF \\
\hline PF & - & $\mathrm{PF}$ & - & $\mathrm{C}$ & PF & - \\
\hline- & $\mathrm{PF}$ & $\mathrm{PF}$ & $\mathrm{PF}$ & - & PF & - \\
\hline- & - & $\mathrm{R}$ & - & - & - & - \\
\hline- & $\mathrm{R}$ & - & $\mathrm{PF}$ & $\mathrm{PF}$ & - & $\mathrm{C}$ \\
\hline- & - & $\mathrm{PF}$ & - & - & - & - \\
\hline- & - & - & - & $\mathrm{PF}$ & PF & $\mathrm{PF}$ \\
\hline- & - & - & $\mathrm{PF}$ & - & - & - \\
\hline- & - & - & $\mathrm{PF}$ & $\mathrm{PF}$ & $\mathrm{PF}$ & - \\
\hline $\mathrm{PF}$ & $\mathrm{PF}$ & $\mathrm{C}$ & $\mathrm{PF}$ & $\mathrm{C}$ & $\mathrm{PF}$ & PF \\
\hline $\mathrm{PF}$ & - & $\mathrm{PF}$ & $\mathrm{PF}$ & $\mathrm{C}$ & PF & PF \\
\hline- & $\mathrm{R}$ & - & - & - & $\mathrm{PF}$ & - \\
\hline $\mathrm{N}$ & $\mathrm{N}$ & A & $\mathrm{C}$ & $\mathrm{D}$ & $\mathrm{N}$ & $\mathrm{N}$ \\
\hline- & - & $\mathrm{PF}$ & - & $\mathrm{PF}$ & - & - \\
\hline PF & $\mathrm{PF}$ & $\mathrm{PF}$ & $\mathrm{C}$ & $\mathrm{N}$ & - & - \\
\hline
\end{tabular}

$\begin{array}{llllll}\mathrm{PF} & \mathrm{R} & \mathrm{PF} & \mathrm{PF} & \mathrm{PF} & -\end{array}$

$\begin{array}{llllllll}- & - & - & - & - & \mathrm{PF} & \mathrm{PF}\end{array}$

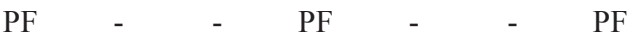

$\begin{array}{ccccccc}- & - & - & - & \mathrm{PF} & - & - \\ - & - & \mathrm{PF} & - & - & \mathrm{PF} & - \\ - & - & \mathrm{R} & \mathrm{PF} & \mathrm{PF} & - & \mathrm{PF} \\ - & - & - & \mathrm{PF} & \mathrm{PF} & \mathrm{C} & \mathrm{PF} \\ \mathrm{PF} & \mathrm{PF} & - & - & - & - & - \\ - & - & \mathrm{PF} & - & - & - & - \\ - & \mathrm{PF} & - & - & \mathrm{PF} & \mathrm{C} & \mathrm{C} \\ - & \mathrm{R} & - & - & - & - & - \\ - & \mathrm{C} & \mathrm{C} & - & - & \mathrm{N} & \mathrm{C} \\ - & - & - & - & \mathrm{C} & \mathrm{PF} & \mathrm{PF} \\ \mathrm{R} & - & - & - & - & - & -\end{array}$




\begin{tabular}{|c|c|c|c|c|c|c|c|}
\hline DIAS DE COLONIZAÇÃO & $3^{\circ}$ & $6^{0}$ & $9^{\circ}$ & $12^{\circ}$ & $15^{\circ}$ & $18^{\circ}$ & $21^{\circ}$ \\
\hline Desmodesmus armatus (Chod.) Hegew. & - & - & $\mathrm{PF}$ & $\mathrm{PF}$ & $\mathrm{PF}$ & $\mathrm{C}$ & $\mathrm{PF}$ \\
\hline $\begin{array}{l}\text { Desmodesmus armatus (Chod.) Hegew. var. bicaudatus (Gugl.) } \\
\text { Hegew. }\end{array}$ & $\mathrm{PF}$ & $\mathrm{PF}$ & - & - & $\mathrm{PF}$ & $\mathrm{PF}$ & - \\
\hline Desmodesmus denticulatus (Lagerh.) Friedl \& Hegew. & - & - & - & $\mathrm{C}$ & $\mathrm{C}$ & $\mathrm{C}$ & $\mathrm{C}$ \\
\hline $\begin{array}{l}\text { Desmodesmus denticulatus (Lagerh.) Friedl \& Hegew. var. } \\
\text { linearis (Hansg.) Hegew. }\end{array}$ & $\mathrm{C}$ & $\mathrm{PF}$ & PF & - & - & - & - \\
\hline Desmodesmus perforatus (Lemm.) Hegew. & $\mathrm{R}$ & - & $\mathrm{PF}$ & $\mathrm{PF}$ & $\mathrm{C}$ & $\mathrm{C}$ & $\mathrm{C}$ \\
\hline Desmodesmus quadricauda (Turp.) Hegew. & $\mathrm{C}$ & $\mathrm{C}$ & $\mathrm{C}$ & $\mathrm{C}$ & $\mathrm{C}$ & $\mathrm{N}$ & $\mathrm{C}$ \\
\hline Desmodesmus serratus (Corda) Friedl \& Hegew. & - & - & $\mathrm{R}$ & - & - & - & - \\
\hline Desmodesmus spinosus (Chod.) Hegew. & - & $\mathrm{PF}$ & $\mathrm{C}$ & $\mathrm{C}$ & $\mathrm{C}$ & $\mathrm{N}$ & - \\
\hline Kirchneriella obesa (West) West \& G.S.West & - & $\mathrm{PF}$ & - & - & - & - & - \\
\hline Monoraphidium arcuatum (Korsh.) Hind. & - & - & - & - & - & $\mathrm{PF}$ & - \\
\hline Monoraphidium caribeum Hind. & - & - & - & - & $\mathrm{C}$ & - & - \\
\hline Monoraphidium cf. circinale (Nyg.) Nyg. & - & - & $\mathrm{PF}$ & - & - & - & - \\
\hline Monoraphidium sp. & $\mathrm{PF}$ & - & - & $\mathrm{PF}$ & - & - & - \\
\hline Oocystis lacustris Chod. & - & - & $\mathrm{PF}$ & - & - & - & - \\
\hline Pediastrum duplex Meyen & - & $\mathrm{R}$ & - & - & - & - & - \\
\hline Pediastrum tetras (Ehr.) Ralfs & - & - & - & - & $\mathrm{PF}$ & - & - \\
\hline Radiococcus sp. & $\mathrm{C}$ & - & $\mathrm{C}$ & - & - & - & - \\
\hline Scenedesmus acuminatus (Lagerh.) Chod. & - & $\mathrm{PF}$ & $\mathrm{PF}$ & - & $\mathrm{PF}$ & $\mathrm{N}$ & $\mathrm{PF}$ \\
\hline Scenedesmus acutus (Meyen) Chod. & - & - & $\mathrm{PF}$ & $\mathrm{C}$ & $\mathrm{C}$ & $\mathrm{C}$ & $\mathrm{PF}$ \\
\hline Scenedesmus acutus (Meyen) Chod. var. alternans Hortob. & - & $\mathrm{R}$ & - & - & - & - & $\mathrm{PF}$ \\
\hline Scenedesmus acutus (Meyen) Chod. var. globosus Hortob. & - & - & - & - & - & $\mathrm{C}$ & $\mathrm{C}$ \\
\hline Scenedesmus cf. antennatus Bréb. & - & - & $\mathrm{PF}$ & - & - & - & - \\
\hline Scenedesmus bijugus (Turp.) Kütz. & $\mathrm{PF}$ & - & $\mathrm{PF}$ & $\mathrm{PF}$ & PF & $\mathrm{C}$ & $\mathrm{PF}$ \\
\hline Scenedesmus javanensis Chod. & $\mathrm{R}$ & - & - & - & - & - & - \\
\hline Scenedesmus sp. & $\mathrm{PF}$ & $\mathrm{N}$ & $\mathrm{N}$ & $\mathrm{PF}$ & $\mathrm{C}$ & $\mathrm{C}$ & $\mathrm{C}$ \\
\hline Selenastrum gracile (Rein.) & $\mathrm{R}$ & $\mathrm{R}$ & - & - & PF & - & - \\
\hline Stigeoclonium sp. & - & $\mathrm{PF}$ & $\mathrm{C}$ & $\mathrm{N}$ & $\mathrm{N}$ & $\mathrm{N}$ & $\mathrm{N}$ \\
\hline Westella $\mathrm{sp}$ & - & - & - & - & $\mathrm{PF}$ & $\mathrm{C}$ & - \\
\hline Chlorococcales não identificada 1 & $\mathrm{PF}$ & $\mathrm{C}$ & - & - & - & - & - \\
\hline Chlorococcales não identificada 2 & - & - & - & - & $\mathrm{C}$ & $\mathrm{C}$ & $\mathrm{C}$ \\
\hline
\end{tabular}




\section{DIAS DE COLONIZAÇÃO}

Chlorococcales não identificada 3

Chlorococcales não identificada 4

Chrysophyceae

Dinobryon cf. sertularia Ehr.

Mallomonas sp.

Salpingoeca marsoni Lemm.

Salpingoeca urceolata kent

Salpingoeca sp.

Synura sp.

Cryptophyceae

Cryptomonas sp.

Cyanophyceae

Anabaena sp.

Aphanocapsa sp.

ChaDesiphon incrustans Grun.

Chroococcus minimus (Keis.) Lemm.

Chroococcus minor (Kütz.) Näg.

Chroococcus sp.

Geitlerinema splendidum (Grev.) Anag.

Gloeocapsa sp.

Jaaginema quadripunctulatum (Brühl. \& Bisw.) Anag. \& Kom. PF

Komvophoron crassum (Voz.) Anag. \& Kom.

Komvophoron minutum (Skuja) Anag. \& Kom.

Leptolyngbya lagerheimii (Gom.) Anag. \& Kom.

Lyngbya comperei Senna

Lyngbya maior Men.

Lyngbya sp.

Merismopedia tenuissima Lemm.

Microcystis aeruginosa (Kütz.) Lemm.

Oscillatoria principes Vaucher

$\begin{array}{lllllll}3^{\circ} & 6^{\circ} & 9^{\circ} & 12^{\circ} & 15^{\circ} & 18^{\circ} & 21^{\circ}\end{array}$

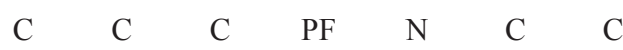

$\begin{array}{llllll}- & \text { D } & \text { A } & \text { A } & \text { D } & \text { D }\end{array}$

$\begin{array}{ccccccc}- & - & \mathrm{R} & - & - & - & - \\ - & - & \mathrm{PF} & - & \mathrm{PF} & - & - \\ - & \mathrm{C} & - & - & - & - & - \\ - & - & - & - & - & - & \mathrm{PF} \\ - & - & \mathrm{C} & \mathrm{N} & \mathrm{N} & - & \mathrm{C}\end{array}$

$\begin{array}{llllllll}\mathrm{C} & \mathrm{PF} & \mathrm{PF} & \mathrm{C} & \mathrm{C} & \mathrm{C} & \mathrm{C}\end{array}$

$\begin{array}{ccccccc}- & \text { R } & \text { PF } & \text { PF } & \text { PF } & \text { PF } & - \\ - & - & - & - & \text { C } & \text { N } & \text { C } \\ - & - & - & - & - & \text { PF } & -\end{array}$

$\begin{array}{lllllll}\text { PF } & \text { C } & \text { PF } & \text { C } & \text { A } & \text { A } & \text { N }\end{array}$

- PF - PF C C PF

$\begin{array}{lllllll}\mathrm{C} & \mathrm{PF} & \mathrm{N} & - & - & - & -\end{array}$

$\begin{array}{lllllll}\mathrm{C} & \mathrm{PF} & \mathrm{C} & \mathrm{C} & \mathrm{N} & \mathrm{C} & \mathrm{C}\end{array}$

$\begin{array}{llllll}- & \mathrm{PF} & - & - & - & -\end{array}$

C C D C

$\begin{array}{lllllll}- & - & - & - & \mathrm{C} & \mathrm{N} & \mathrm{N}\end{array}$

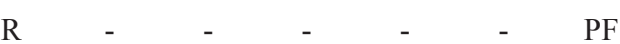

$\begin{array}{llllll}- & \mathrm{C} & \mathrm{C} & \mathrm{PF} & \mathrm{N} & -\end{array}$

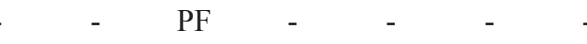

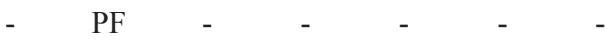

$\begin{array}{llllllll}- & - & - & - & - & - & - & \end{array}$

$\begin{array}{lllllllllllllll}\mathrm{R} & \mathrm{C} & - & - & \mathrm{C} & \mathrm{PF} & \mathrm{PF}\end{array}$

PF PF




\section{DIAS DE COLONIZAÇÃO}

Phormidium chlorinum (Kütz.) Umez. \& Wat.

Phormidium irriguum (Kütz. ex Gom.) Anag. \& Kom.

Planktolyngbya limnetica (Lemm.) Kom.-Legn.

Planktolyngbya sp.

Planktothrix sp.

Pseudanabaena catenata Laut.

Pseudanabaena lonchoides Anag.

Pseudanabaena moniliformes Kom. \& Kling

Pseudanabaena mucicula (Hub.-Pest. \& Naum.) Schw.

Raciborskia sp.

Rhabdoderma vermiculare Fott

Snowella sp.

Chroococcales não identificada

Dinophyceae

Peridinium cf. volzii Lemm.

Euglenophyceae

Euglena sp.

Phacus sp.

Trachelomonas hispida (Perty) Stein.

Bulbochaete sp. 1

Bulbochaete sp. 2

Bulbochaete sp. 4

Bulbochaete sp. 5

Oedogonium sp.

Oedogonium sp. 1

Oedogonium sp. 3

Oedogonium sp. 4

Oedogonium sp. 5

Ulothricophyceae

Ulothrix sp.

$\begin{array}{ccccccc}3^{\mathbf{0}} & \mathbf{6}^{\mathbf{0}} & \mathbf{9}^{\mathbf{0}} & \mathbf{1 2}^{\mathbf{0}} & \mathbf{1 5}^{\mathbf{0}} & \mathbf{1 8}^{\mathbf{0}} & \mathbf{2 1}^{\mathbf{0}} \\ \mathrm{C} & \mathrm{C} & \mathrm{N} & \mathrm{C} & \mathrm{C} & \mathrm{C} & \mathrm{PF} \\ \mathrm{N} & - & - & \mathrm{C} & - & - & - \\ - & - & \mathrm{C} & - & - & - & - \\ - & - & - & - & \mathrm{C} & - & - \\ \mathrm{N} & \mathrm{A} & \mathrm{N} & \mathrm{D} & \mathrm{D} & \mathrm{D} & \mathrm{D} \\ - & - & - & \mathrm{C} & \mathrm{PF} & \mathrm{C} & \mathrm{C} \\ \mathrm{PF} & - & - & \mathrm{C} & - & - & - \\ \mathrm{PF} & \mathrm{PF} & - & \mathrm{C} & - & \mathrm{N} & \mathrm{PF} \\ - & \mathrm{PF} & - & - & - & - & - \\ - & - & - & - & - & \mathrm{PF} & - \\ \mathrm{R} & \mathrm{PF} & \mathrm{PF} & - & \mathrm{PF} & \mathrm{PF} & - \\ - & - & \mathrm{PF} & - & - & - & -\end{array}$

PF PF $\quad-\quad-\quad-\quad-\quad P F \quad P F$

- $\mathrm{R} \quad-\quad-\mathrm{PF} \quad-\quad \mathrm{PF}$

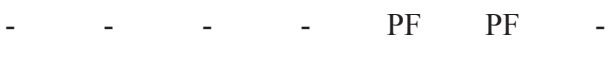

$\begin{array}{lllllll}- & - & P F & - & - & - & -\end{array}$

$\begin{array}{llllll}- & - & - & P F & C & P F\end{array}$

- $\quad$ - $\quad$ - $\quad$ - $\quad$ PF PF PF

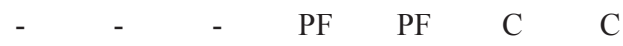

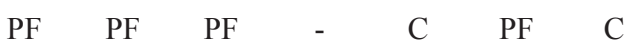

$\begin{array}{lllllll}- & \mathrm{C} & \mathrm{N} & \mathrm{C} & \mathrm{C} & \mathrm{N} & \mathrm{N}\end{array}$

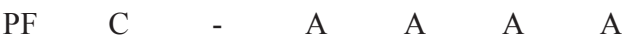

$\begin{array}{llllll}- & \mathrm{PF} & - & - & - & -\end{array}$

$\begin{array}{lllllll}\text { PF } & \mathrm{N} & \mathrm{N} & \mathrm{N} & \mathrm{A} & \mathrm{D} & \mathrm{A}\end{array}$ 


\section{DIAS DE COLONIZAÇÃO}

Uronema elongatum Hodg.

Uronema sp.

Xanthophyceae

Characiopsis aquilonaris Skuja

Characiopsis sphagnicola Pascher

Stipitococcus vasiformis Tiff.

Zygnemaphyceae

Actinotaenium globosum (Bulnh.) Teil.

Closterium acutum Ehr.

Closterium closterioides (Ralfs) Louis \& Peet.

Closterium closterioides var. intermedium (Roy \& Biss.) Ruz.

Closterium cornu Ehr. ex Ralfs

Closterium incurvum Bréb.

Closterium jenneri Ralfs

Closterium cf. leibleinii Kütz. ex Ralfs

Closterium moniliferum (Bory) Ehr. ex Ralfs

Closterium navicula (Bréb.) Lütk.

Closterium cf. venus Kütz. ex Ralfs

Closterium sp.

Cosmarium abbreviatum Racib. var. minus (West \& G. S. West) W. Krieg. \& Gerl.

Cosmarium blytty Wille

Cosmarium brasiliense (Wille) Nordst.

Cosmarium comissurale (Bréb.) Ralfs var. crassum Nordst.

Cosmarium contractum Kirch.

Cosmarium excavatum Nordst.

Cosmarium granatum Bréb. ex Ralfs

Cosmarium laeve Rabenh.

Cosmarium exiguum Archer var. pressum West \& West

Cosmarium cf. moerlianum Lütk. var. brasiliense Borge

Cosmarium naegelianum Bréb.

$3^{\circ} \quad 6^{\circ}$

$9^{\circ}$

$12^{\circ}$

$15^{\circ}$

$18^{\circ}$

$21^{\circ}$

PF

$\begin{array}{ccccccc}- & \mathrm{R} & \mathrm{R} & \mathrm{PF} & \mathrm{C} & \mathrm{N} & \mathrm{C} \\ \mathrm{PF} & - & - & - & - & - & - \\ - & \mathrm{PF} & - & - & - & - & -\end{array}$

$\begin{array}{cccccc}\text { R } & \text { PF } & \text { PF } & \text { PF } & \text { C } & \text { PF } \\ \text { PF } & \text { PF } & - & - & - & -\end{array}$

$\begin{array}{lllll}- & - & - & - & \\ - & - & - & - & \end{array}$

PF PF PF $\quad-\quad$ - PF PF

$\mathrm{PF} \quad-\quad \mathrm{PF} \quad-\quad \mathrm{PF}$

$\mathrm{PF} \quad-\quad \mathrm{PF}$

- $\quad-\quad$ PF $\quad$ C $\quad$ C

- $\quad \mathrm{PF} \quad-\quad$ - $\quad$ - $\quad$ PF

$\begin{array}{llllll}\text { R } & \mathrm{R} & \mathrm{PF} & \mathrm{PF} & \mathrm{PF} & \mathrm{PF}\end{array}$

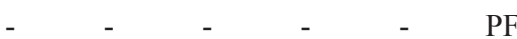

$-\quad P$

$\begin{array}{lllllll}\mathrm{C} & \mathrm{C} & \mathrm{N} & \mathrm{PF} & \mathrm{C} & \mathrm{C} & \mathrm{PF}\end{array}$

$\begin{array}{llllllllllllll}- & - & \mathrm{PF} & - & - & - & - & -\end{array}$

- $\quad$ - $\quad$ PF PF PF PF PF

- $\quad-\quad-\quad-\quad P F$

$\mathrm{PF} \quad-\quad \mathrm{C} \quad-\quad \mathrm{PF}$

$\begin{array}{llllll}- & - & \mathrm{PF} & - & - & -\end{array}$

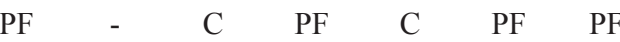

$\begin{array}{lllllll}\mathrm{C} & \mathrm{PF} & \mathrm{C} & \mathrm{C} & \mathrm{C} & \mathrm{C} & \mathrm{PF}\end{array}$

$\begin{array}{lllllll}- & \mathrm{C} & \mathrm{N} & \mathrm{N} & \mathrm{C} & \mathrm{N} & \mathrm{N}\end{array}$

$\begin{array}{llllllll}- & - & - & - & - & - & - & \end{array}$

$\begin{array}{llllllll}\mathrm{C} & \mathrm{C} & \mathrm{C} & \mathrm{C} & \mathrm{C} & \mathrm{PF} & -\end{array}$ 


\section{DIAS DE COLONIZAÇÃO}

Cosmarium norimbergense Reinsch var. depressum (West \& G. S. West) W. Krieg. \& Gerl.

Cosmarium cf. phaseolus var. phaseolus Bréb. ex Ralfs f. minus Boldt.

Cosmarium portianum Arch.

Cosmarium pseudoconnatum Nordst.

Cosmarium pseudoexigum Racib.

Cosmarium punctulatum Bréb.

Cosmarium regnesii Reinsch

Cosmarium reniforme (Ralfs) Arch.

Cosmarium reniforme (Ralfs) Arch. forma menor

Cosmarium sublobatum (Bréb.) Arch. var. brasiliense Borge

Cosmarium subspeciosum Nordst.

Cosmarium subtumidum Nordst.

Cosmarium trilobulatum Reinsch

Cosmarium cf. vexatum $\mathrm{W}$. West

Cosmarium sp.

Cylindrocystis brebisonnii (Menegh. ex Ralfs) de Bary

Euastrum abruptrum Nordst.

Euastrum denticulatum (Kirchn.) Gay

Euastrum monocylum (Nordst.) Racib.

Gonatozygon monotaenium de Bary

Gonatozygon pilosum Wolle

Hyalotheca dissiliens (Smith) Bréb. ex Ralfs

Micrasterias abrupta West \& G.S. West

Micrasterias furcata Ralfs

Micrasterias truncata (Corda) Bréb. ex Ralfs var. pusilla G.S. West

Mougeotia sp.

Mougeotia sp. 1

Mougeotia sp. 2

Mougeotia sp. 3

Mougeotia sp. 4

\section{$3^{\circ}$}

$6^{\circ}$

$\mathbf{9}^{\mathbf{9}}$

12

$15^{\circ}$

$18^{\circ}$

$21^{\circ}$

$\begin{array}{ccccccc}- & - & - & \text { PF } & \text { PF } & \text { PF } & - \\ \text { R } & \text { PF } & \text { N } & \text { C } & \text { C } & \text { C } & \text { C }\end{array}$

- $\quad$ PF

$\begin{array}{llllll}\mathrm{PF} & \mathrm{PF} & \mathrm{PF} & \mathrm{PF} & \mathrm{C} & \mathrm{PF}\end{array}$

- $\quad$ - $P F \quad-\quad P F \quad C \quad P F$

$\begin{array}{llllll}\mathrm{C} & \mathrm{C} & \mathrm{N} & \mathrm{PF} & \mathrm{C} & \mathrm{C}\end{array}$

$\begin{array}{lllllll}\mathrm{C} & \mathrm{C} & \mathrm{A} & \mathrm{C} & \mathrm{N} & \mathrm{C} & \mathrm{PF}\end{array}$

$\begin{array}{llllll}- & \text { PF } & \text { PF } & \text { PF } & \text { C } & \text { C }\end{array}$

- $\quad$ - $\quad$ - $\quad$ PF PF

$-$

- $\quad$ - $\quad$ PF - PF

$\begin{array}{llllll}C & \text { PF } & \text { PF } & \text { PF } & \text { PF } & \text { PF }\end{array}$

$\begin{array}{lllllll}\text { PF } & \text { C } & \text { PF } & \text { C } & \text { C } & \text { PF } & \text { PF }\end{array}$

- $\quad$ - $\quad-\quad$ - $\quad$ PF

$\begin{array}{llllllll}\mathrm{PF} & \mathrm{C} & \mathrm{C} & \mathrm{C} & \mathrm{C} & \mathrm{PF} & \mathrm{C}\end{array}$

$\begin{array}{llllll}- & - & - & - & P F & P F\end{array}$

$\begin{array}{llllll}- & \mathrm{R} & - & \mathrm{PF} & \mathrm{PF} & \mathrm{PF}\end{array}$

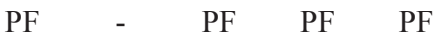

- $\quad$ - PF $\quad-\quad$ - $\quad-$

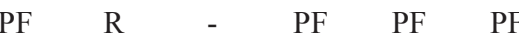

-
-

$\begin{array}{lllllll}\mathrm{R} & \mathrm{PF} & \mathrm{PF} & \mathrm{PF} & \mathrm{C} & \mathrm{C}\end{array}$

- $\quad-\quad \mathrm{R} \quad-\quad-\mathrm{C}_{-}$

- $\quad$ - PF - PF -

$\begin{array}{ccccccc}- & \mathrm{R} & - & - & - & - & - \\ - & \mathrm{C} & \mathrm{C} & \mathrm{C} & \mathrm{N} & \mathrm{PF} & \mathrm{PF} \\ \mathrm{PF} & - & - & - & \mathrm{N} & \mathrm{C} & \mathrm{C} \\ \mathrm{PF} & \mathrm{C} & \mathrm{C} & \mathrm{PF} & \mathrm{N} & \mathrm{PF} & \mathrm{PF} \\ - & - & - & - & \mathrm{PF} & - & - \\ - & - & - & \mathrm{PF} & - & - & \mathrm{PF}\end{array}$




\section{DIAS DE COLONIZAÇÃO}

Netrium oblongum (De Bary) Lütk.

Netrium digitus (Ehr.) Itzigs. \& Rothe

Penium sp.

Sphaerozosma aubertianum W. West

Spirogyra sp.

Spirogyra sp. 1

Spondylosium moniliforme Lund.

Spondylosium planum (Wolle) West \& G.S. West

Spondylosium pulchrum Bail.

Staurastrum ambiguum Turner

Staurastrum anatinum Cooke \& Wills

Staurastrum boreale West \& G.S. West

Staurastrum brebissoni Arch. var. brasiliense Grönbl.

Staurastrum claviferum West \& G.S. West

Staurastrum cf. cyclacanthum West \& G.S. West

Staurastrum dilatatum (Ehr.) Ralfs

Staurastrum hagmannii Grönbl.

Staurastrum hantzschii Reinsch

Staurastrum leptocladum Nordst.

Staurastrum margaritaceum (Ehr.) Ralfs

Staurastrum muticum (Bréb.) Ralfs

Staurastrum nudibrachiatum Borge

Staurastrum orbiculare (Ehr.) Ralfs

Staurastrum orbiculare var. depressum Roy \& Biss.

Staurastrum cf. polymorphum Bréb.

Staurastrum quadricornutum Roy \& Biss.

Staurastrum quadrangulare Bréb. ex Ralfs var. var. quadrangulare

Staurastrum quadrangulare var. contectum (Turn.)

Staurastrum sebaldi Reinsch var. ornatum Nordst.

Staurastrum minesotense Wolle

\begin{tabular}{|c|c|c|c|c|c|c|}
\hline $3^{0}$ & $6^{0}$ & $9^{\circ}$ & $12^{\circ}$ & $15^{\circ}$ & $18^{\circ}$ & $21^{\circ}$ \\
\hline- & $\mathrm{PF}$ & - & - & - & - & - \\
\hline - & $\mathrm{PF}$ & - & - & $\mathrm{PF}$ & - & - \\
\hline- & - & - & - & $\mathrm{PF}$ & - & - \\
\hline $\mathrm{R}$ & - & - & - & - & - & - \\
\hline $\mathrm{PF}$ & $\mathrm{R}$ & $\mathrm{PF}$ & $\mathrm{PF}$ & $\mathrm{N}$ & $\mathrm{PF}$ & $\mathrm{PF}$ \\
\hline- & - & - & - & - & $\mathrm{PF}$ & $\mathrm{PF}$ \\
\hline $\mathrm{PF}$ & $\mathrm{PF}$ & - & $\mathrm{PF}$ & - & - & - \\
\hline - & $\mathrm{R}$ & $\mathrm{PF}$ & $\mathrm{PF}$ & $\mathrm{C}$ & $\mathrm{PF}$ & - \\
\hline- & $\mathrm{R}$ & - & - & - & - & - \\
\hline $\mathrm{PF}$ & $\mathrm{PF}$ & - & - & $\mathrm{PF}$ & - & $\mathrm{PF}$ \\
\hline $\mathrm{PF}$ & $\mathrm{R}$ & $\mathrm{PF}$ & $\mathrm{PF}$ & - & - & - \\
\hline - & - & $\mathrm{PF}$ & - & - & - & - \\
\hline $\mathrm{R}$ & $\mathrm{R}$ & - & - & - & - & - \\
\hline- & $\mathrm{PF}$ & $\mathrm{PF}$ & $\mathrm{PF}$ & $\mathrm{C}$ & $\mathrm{PF}$ & - \\
\hline- & - & - & - & $\mathrm{C}$ & - & - \\
\hline $\mathrm{PF}$ & C & C & - & $\mathrm{PF}$ & $\mathrm{PF}$ & - \\
\hline- & $\mathrm{PF}$ & $\mathrm{PF}$ & $\mathrm{PF}$ & $\mathrm{PF}$ & $\mathrm{PF}$ & - \\
\hline $\mathrm{PF}$ & PF & $\mathrm{R}$ & - & - & - & - \\
\hline $\mathrm{PF}$ & - & - & - & $\mathrm{PF}$ & - & - \\
\hline $\mathrm{C}$ & $\mathrm{C}$ & $\mathrm{C}$ & - & $\mathrm{PF}$ & $\mathrm{PF}$ & - \\
\hline- & - & $\mathrm{C}$ & - & $\mathrm{PF}$ & $\mathrm{PF}$ & - \\
\hline- & $\mathrm{PF}$ & - & - & $\mathrm{PF}$ & - & - \\
\hline- & $\mathrm{PF}$ & - & - & - & - & - \\
\hline- & $\mathrm{PF}$ & $\mathrm{PF}$ & - & $\mathrm{PF}$ & - & - \\
\hline- & $\mathrm{R}$ & - & - & - & - & - \\
\hline $\mathrm{C}$ & C & C & PF & $\mathrm{PF}$ & - & - \\
\hline $\mathrm{R}$ & $\mathrm{PF}$ & $\mathrm{PF}$ & $\mathrm{PF}$ & $\mathrm{PF}$ & - & $\mathrm{PF}$ \\
\hline $\mathrm{PF}$ & - & - & - & $\mathrm{PF}$ & - & - \\
\hline $\mathrm{C}$ & $\mathrm{PF}$ & $\mathrm{PF}$ & - & - & $\mathrm{C}$ & - \\
\hline PF & - & - & - & - & - & - \\
\hline
\end{tabular}




\begin{tabular}{|c|c|c|c|c|c|c|c|}
\hline DIAS DE COLONIZAÇÃO & $3^{\circ}$ & $6^{\mathbf{0}}$ & $9^{\circ}$ & $12^{\circ}$ & $15^{\circ}$ & $18^{\circ}$ & $21^{\circ}$ \\
\hline Staurastrum setigerum Cleve var. pectinatum West \& G.S. West & $\mathrm{PF}$ & $\mathrm{R}$ & $\mathrm{PF}$ & - & $\mathrm{PF}$ & - & - \\
\hline Staurastrum tetracerum (Kütz.) Ralfs & $\mathrm{R}$ & $\mathrm{PF}$ & $\mathrm{C}$ & $\mathrm{C}$ & $\mathrm{C}$ & $\mathrm{C}$ & - \\
\hline Staurastrum trifidium Nordst. var. inflexum West \& G.S. West & $\mathrm{PF}$ & $\mathrm{PF}$ & $\mathrm{PF}$ & $\mathrm{PF}$ & $\mathrm{PF}$ & $\mathrm{PF}$ & - \\
\hline Staurastrum sp. & - & $\mathrm{PF}$ & $\mathrm{PF}$ & $\mathrm{PF}$ & $\mathrm{PF}$ & - & - \\
\hline Staurodesmus brevispina (Bréb.) Croas. & - & - & $\mathrm{PF}$ & - & - & - & - \\
\hline Staurodesmus corniculatus (Lund.) Teil. var. spinigerum W. West & - & - & $\mathrm{PF}$ & $\mathrm{PF}$ & $\mathrm{PF}$ & - & - \\
\hline Staurodesmus cuspidatus (Bréb. ex Ralfs) Teil. & $\mathrm{PF}$ & - & $\mathrm{PF}$ & - & $\mathrm{PF}$ & $\mathrm{PF}$ & - \\
\hline Staurodesmus dejectus (Bréb.) Teil. & - & $\mathrm{PF}$ & - & - & $\mathrm{PF}$ & - & - \\
\hline Staurodesmus dejectus var. brevispinus (Nygaa.) Coesel & $\mathrm{PF}$ & - & - & - & - & - & - \\
\hline Staurodesmus dickiei (Ralfs) S. Lill. & - & - & $\mathrm{C}$ & $\mathrm{PF}$ & $\mathrm{PF}$ & - & - \\
\hline Staurodesmus glaber (Ehr. ex Ralfs) S. Lill. & - & - & $\mathrm{PF}$ & - & $\mathrm{PF}$ & - & - \\
\hline Staurodesmus mamillatus (Nordst.) Teil. & $\mathrm{R}$ & $\mathrm{R}$ & - & - & $\mathrm{C}$ & $\mathrm{PF}$ & $\mathrm{PF}$ \\
\hline Staurodesmus patens (Nordst.) Croas. & - & - & $\mathrm{PF}$ & - & - & - & - \\
\hline Staurodesmus cf. spencerianus (Dsk.) Teil. & - & $\mathrm{PF}$ & - & - & - & - & - \\
\hline Staurodesmus sp. & $\mathrm{PF}$ & $\mathrm{R}$ & - & - & - & $\mathrm{PF}$ & - \\
\hline Teilingia granulata (Roy et Biss.) Bourr. & - & - & - & - & $\mathrm{PF}$ & - & - \\
\hline Zygnema sp. & - & - & $\mathrm{PF}$ & - & $\mathrm{PF}$ & - & - \\
\hline Desmidiaceae não identificada & $\mathrm{PF}$ & $\mathrm{C}$ & $\mathrm{R}$ & $\mathrm{PF}$ & $\mathrm{PF}$ & - & - \\
\hline
\end{tabular}

